\title{
ESTUDIO GOMPARADO ENTRE ESPAÑA Y MÉXICO SOBRE EL MARCO JURÍDICO APLICABLE AL FEMINICIDIO*
}

\author{
A COMPARATIVE STUDY BETWEEN THE SPANISH AND MEXICAN \\ LAWS REGARDING FEMICIDE
}

\section{Carmen MONTERO**}

RESUMEN: La idea de llevar a cabo el presente trabajo surge a raíz de los casos de feminicidios que en los últimos años están teniendo lugar en España y la consecuente necesidad de que el gobierno tome todas las medidas posibles para atajar la comisión de los actos delictivos que causan el asesinato de mujeres, empezando por la tipificación del feminicidio como un delito autónomo en el Código Penal español. Al hilo de estas circunstancias en este trabajo, se realiza un análisis de las causas que dan lugar a la comisión de tales actos, se argumenta la necesidad de su tipificación y se proponen algunas estrategias para erradicar la violencia feminicida. Asimismo, estos mismos parámetros analíticos (causas, tipificación y estrategias para su erradicación) se utilizan para estudiar la cuestión de los feminicidios en México.

Palabras clave: feminicidio, tipificación penal, España, México.

\begin{abstract}
The idea of putting this paper together arises from the recent several cases of feminicide in Spain, and the resulting need for the Spanish government to take all affordable measures to attend the committing of gender crimes which cause the murder of women. The first step should be to classify the feminicide in the Spanish Criminal Code as a separate offense. Based on these circumstances, this paper analysis on the reasons underlaying the committing of this kind of acts, an argumentation on the need to classify them as criminal offenses, and a proposal of some strategies to eradicate the feminicide violence. Likewise, these aforementioned analytical parameters (reasons, classification and strategies for eradication) are used to study the feminicide issue in Mexico.
\end{abstract}

Keyzoords: Feminicide, Criminal Classification, Spain, Mexico.

* Artículo recibido el 25 de abril de 2016 y aceptado para su publicación el 7 de junio de 2017.

** ORCID: 0000-0002-0060-3712. Investigadora posdoctoral en Université Laval (Canadá), 2018-2020. Becaria de la Xunta de Galicia (Convocatoria de axudas de apoio á etapa de formación posdoutoral nas universidades do SUG, nos organismos públicos de investigación de Galicia, Modalidad A) y miembro del Grupo de Estudios Internacionales de la Universidad de Santiago de Compostela (GRESIN-GI-1138). Correo electrónico: carmenmonteroferrer@gmail.com.

Boletín Mexicano de Derecho Comparado, nueva serie, año LI, núm. 154, enero-abril de 2019, pp. 147-170.

Esta obra está bajo una Licencia Creative Commons Atribución-NoComercial-SinDerivar 4.0 Internacional, IIJ-UNAM. 
SUMARIO. I. Introducción. II. Consideraciones previas. III. Las causas de la violencia feminicida: ¿por qué tipificar el feminicidio? IV. Marco jurídico aplicable y estrategias nacionales para su erradicación. V. Conclusiones. VI. Bibliografia.

\section{INTRODUCGIÓN}

Más de 99, ésa es la cifra de asesinatos y feminicidios cometidos contra mujeres en España a lo largo de 2017; junto a ellas 6 varones fallecieron, víctimas de violencia machista, en el mismo periodo. Estos datos dan muestra, sin lugar a duda, de que España está experimentando un pico de feminicidios y asesinatos de mujeres, por lo que consideramos que es absolutamente necesario revisar el debate jurídico en torno a esta problemática. En este sentido, cabe preguntarse acerca del estado de la situación en España y las soluciones propuestas por el marco jurídico actual. Asimismo, en este trabajo se plantea la hipótesis de la conveniencia de adoptar un tipo específico de feminicidio en el ámbito jurídico penal español, y se efectúan una serie de recomendaciones que consideramos que, al hilo del informe presentado por el Grupo de Trabajo de las Naciones Unidas de discriminación contra la mujer, presentado en junio de 2015, deberían ser tomadas en cuenta por los poderes públicos nacionales a fin de acabar con este tipo de violencia que tiene como víctimas directas a las mujeres, pero que afecta a toda la sociedad. ${ }^{1}$ Por otra parte, se analizan las causas por las que el feminicidio, a pesar de su tipificación como delito autónomo, sigue siendo un problema estructural en México.

Ante dos contextos tan dispares, cabe plantearse si las causas por las que suceden los actos feminicidas en ambos lados del Atlántico son, a priori, equiparables. En principio consideramos que nos enfrentamos en ambas sociedades a un problema estructural, fruto de la enraizada cultura patriarcal que ha imperado durante siglos tanto en España como en México. Por ello, es pertinente llevar a cabo dicha comparativa entre ambos países a fin de constatar si las realidades entre ambos son tan dispares como pudiese parecer en un primer momento.

A este fin, en un primer epígrafe se van exponer unas breves cuestiones previas que conviene tener en cuenta antes de abordar en profundidad el tema, para centrarnos en un segundo epígrafe en las causas que subya-

1 Organización de Naciones Unidas (ONU), Asamblea General, Consejo de Derechos Humanos. Informe del Grupo de Trabajo en la cuestión de la discriminación contra la mujer en la ley y en la práctica. Adición. Misión a España. A/HRC/29/40/Add.3, 17 de junio de 2015.

Esta obra está bajo una Licencia Creative Commons

Atribución-NoComercial-SinDerivar 4.0 Internacional, IIJ-UNAM.

Boletín Mexicano de Derecho Comparado, núm. 154, enero-abril de 2019, pp. 147-170. 
cen en la comisión de este tipo de actos delictivos; asimismo, a lo largo de este mismo epígrafe se analiza el término "feminicidio" y se esbozan los argumentos por los que se considera necesaria la tipificación de éste como delito autónomo en las legislaciones nacionales; en el tercer epígrafe se estudian separadamente las situaciones de México y España en relación a este hecho delictivo. El trabajo finaliza con la exposición de las conclusiones y el relato de una suerte de recomendaciones que, se considera, debieran ser tomadas en cuenta por los gobiernos español y mexicano para poner fin a los feminicidios en ambos países.

\section{CONSIDERAGIONES PREVIAS}

El término "feminicidio" se introdujo en el debate jurídico a raíz del conocido como caso Campo Algodonero en el que la Corte Interamericana de Derechos Humanos dictó, con fecha 16 de noviembre de 2009, sentencia contra el Estado mexicano señalando, entre otras cosas, que el Estado incumplió su deber de investigar — y con ello su deber de garantizar - los derechos a la vida, integridad personal y libertad personal, en perjuicio de Claudia Ivette González, Laura Berenice Ramos Mónarrez y Esmeralda Herrera Montreal, las cuales fueron brutalmente asesinadas en el municipio mexicano de Ciudad Juárez ${ }^{2}$ (caso González y otras [Campo Algodonero] vs. México, Corte Interamericana de Derechos Humanos [CIDH], 2009).

Así, el término hace referencia a las muertes de mujeres motivadas por el sexismo y la misoginia, que implican el desprecio y odio hacia ellas sobre la base del sentimiento que subyace en la mente criminal, la cual

2 Previamente, la Comisión Interamericana de Derechos Humanos había interpuesto ante la CIDH una demanda contra México en los casos 12.496, 12.497 y 12.498 (correspondientes a las víctimas anteriormente citadas) por denegación de justicia en relación con la desaparición y homicidio de las víctimas; por la falta de políticas de prevención en estos casos pese al conocimiento de las autoridades de la existencia de un patrón de violencia contra las mujeres y niñas en el Estado de Chihuahua; la ausencia de respuesta de las autoridades frente a estas desapariciones; por no actuar con la debida diligencia en la investigación de las desapariciones y por no ofrecer una adecuada reparación a los familiares de las víctimas. Con ello, el Estado mexicano violó los derechos de acceso a la justicia y a la protección judicial consagrados en los artículos 8.1 y 25.1 de la Convención Americana de Derechos Humanos ("Pacto de San José"), del 22 de noviembre de 1969, y asimismo contravino los preceptos de la Convención Interamericana para Prevenir, Sancionar y Erradicar la Violencia contra la Mujer ("Convención Belém do Pará”), del 9 de junio de 1994. 
considera que ostenta el derecho de terminar con las vidas de estas mujeres al suponerse acreedora de un derecho de propiedad sobre ellas (Russell, 2006: 73-98). Por lo tanto, debemos excluir de esta categorización aquellas muertes en las que el sexo y el género de la víctima son irrelevantes para la persona que comete el acto homicida, pues en este caso se trataría de homicidios o asesinatos no feminicidas.

Pese a la existencia de ésta y otras definiciones que gozan de gran aceptación general, y a pesar de que el término "feminicidio" ya ha sido tipificado en catorce países de América Latina, es necesario ahondar en la necesidad de que el feminicidio sea objeto de mayor desarrollo legislativo, tanto en los instrumentos regionales especializados en la materia, Convención de Belém do Pará y Convenio de Estambul, 2011, como en los ordenamientos jurídicos de los países europeos. En consecuencia, la pertinencia de llevar a cabo este análisis resulta del incremento de las muertes violentas de mujeres registradas en el último decenio en ambos países, a la par que se están incorporando observatorios, mecanismos y protocolos destinados a identificar los feminicidios y responder a su comisión a través del establecimiento de instancias político-administrativas destinadas a proteger el derecho de las mujeres a gozar de una vida libre de violencias.

\section{LAS CAUSAS DE LA VIOLENCIA FEMINICIDA: ¿POR QUÉ TIPIFICAR EL FEMINICIDIO?}

En un estudio de 2006, las investigadoras Russell y Radford realizaron un análisis sociológico de las causas que explican y contextualizan los actos feminicidas (que ellas denominan "terrorismo sexual") a lo largo de la historia. Basándonos en dicho estudio, identificamos los siguientes patrones que se repiten en la mayoría de los episodios feminicidas:

\section{Escalada de violencia como parte de la reacción masculina ante el feminismo}

En la Europa de los siglos XVI y XVII, muchas personas - en su gran mayoría mujeres - fueron condenadas a prisión y ejecutadas, acusadas del crimen de hechicería. Este episodio de cacería a las personas acusadas de "brujas", denominado muy acertadamente "brujomanía" (Trevor-Ro- 
per, 1969), constituye según Russell y Radford, un auténtico feminicidio. ${ }^{3}$ El carácter feminicida de este tipo de violencia no se determina por el sexo de las víctimas, a pesar de que el 90\% de víctimas de brujomanía eran mujeres (MacFarlane, 1970), sino que se infiere del hecho de que los asesinatos de estas mujeres constituían un instrumento de control social, cuyo propósito era aplacar la voluntad de las mujeres que habían empezado a incorporarse al mercado laboral como trabajadoras de la industria textil. ${ }^{4}$

Este episodio histórico nos recuerda otro caso semejante que a nuestro entender ilustra la misma idea; pues al margen de las diferencias cronológicas y contextuales existentes entre los dos casos, no podemos dejar pasar el paralelismo existente con los feminicidios de Ciudad Juárez. Dicho municipio, ha experimentado en los últimos años un crecimiento exponencial de su población, debiendo ser repartidos los recursos de vida entre más mano de obra. Si a esto añadimos la importancia de la industria maquiladora en la zona, en la cual trabajan fundamentalmente mujeres (Fernández de Juan, 2004), tenemos un escenario muy similar al que se vivió en la Inglaterra del siglo XVII con la industria textil. Así, algunas autoras apuntan al factor trabajo como fuente de victimización de la mujer, en un entorno en el que se están dando altas tasas de desempleo masculino (Pantaleo, 2010).

3 Así, la brujomanía puede verse como un ejemplo de feminicidio en el que la violencia contra las mujeres por parte de los hombres se usaba como mecanismo de control social, y descansaba en un constructo particular de la sexualidad femenina, asociada con la visión pecaminosa que la Iglesia católica tenía de la mujer, basada en la construcción del Génesis, la cual legitima la subyugación de las mujeres a los hombres con base en la leyenda de Adán y Eva (Russell, 2006; Hester, 2006).

4 En la Inglaterra de los siglos XIV y XV, las industrias de lana y las tejedoras eran una parte muy importante de la economía del país y también resultaron cruciales para el desarrollo del capitalismo. Durante los siglos posteriores, siglos XVI y XVII, las características de la población estaban cambiando, las personas se casaban tarde, como consecuencia de la necesidad de trabajar para poder financiar las bodas, por lo que existía un importante grupo de mujeres solteras que competían con los hombres por los medios de vida. A esto hay que añadir que a mediados del siglo XVI, las mujeres estaban empezando a invadir algunos espacios que hasta entonces habían sido considerados de dominio masculino, como fue el ascenso de las mujeres monarcas en las cortes europeas. Este dominio femenino fue visto como indeseado y contranatura, lo que propició fuertes críticas misóginas y la apertura del debate denominado "Controversia Popular" — en el que se discutía la posibilidad de que las mujeres estuviesen en posición de igualdad a los hombres-, y en el marco del cual se cosecharon las ideas de que las mujeres eran débiles y más proclives al pecado, y por lo tanto más peligrosas ya que, aprovechando sus dotes sexuales podían pactar con el Diablo, lo que las haría más poderosas (Hester, 2006: 84). 
En consecuencia, como señala Linvigston (2004: 59-76) el feminicidio puede ser ocasionado como una respuesta negativa en contra de la mujer que "obtiene una mayor autonomía personal e independencia, mientras que los hombres pierden su condición de género dominante". Posiblemente, esta teoría, que atiende a la incorporación de la mujer al mercado laboral como factor desencadenante de la violencia de género, podría trasladarse a Europa, y especialmente a España, y, en consecuencia, considerarse como causa de la perpetración de actos feminicidas.

\section{La hipermasculinidad y el sentido de propiedad}

Con la idea de que "si las niñas o mujeres los superan, algunos se vuelven violentos, hasta llegar al extremo de cometer feminicidio", las investigadoras Russel y Radford quisieron dar cuenta de otro de los factores que contribuyen a la comisión de feminicidios. Se trataría del sentimiento de ataque a la masculinidad de los agresores, el cual es generado por el hecho de que las mujeres conquisten esferas político-sociales y/o económicas de las que anteriormente sólo eran acreedores los hombres. Como respuesta, algunos hombres - en un giro hacia la hipermasculinización de su persona - se sienten vulnerables y subordinan a las mujeres, a las que consideran de su propiedad (Russell, 2006: 62); hasta el punto de considerarse con el derecho de decidir cuándo y cómo terminar con sus vidas. Sucede así en los mal llamados "crímenes de honor", en los que el perpetrador justifica la muerte de la mujer en el hecho de que se ha mancillado su propio honor (el del hombre) al ser éste el propietario de la sexualidad femenina.

\section{La banalización institucional de la problemática}

Junto a estos factores conviven otras razones ambientales y culturales que propician o, cuanto menos, no previenen, la comisión de actos feminicidas. En este sentido, el machismo y la misoginia que en muchos casos rigen el comportamiento social se trasladan a las instituciones públicas, dando lugar a una mala praxis en la prevención de tales actos, y a la escasez e inoperatividad en su investigación y enjuiciamiento. 


\section{4. ¿Es el feminicidio un tipo especial de homicidio o asesinato?}

Las investigadoras y académicas latinoamericanas, han diferenciado los feminicidios de los homicidios u asesinatos sin motivación de género (Mónarrez Fragoso, 2002: 279-305), al considerar necesaria la utilización de un término que haga referencia a las características específicas que conllevan este tipo de actos, sobre todo en Centroamérica, donde la mayoría de los homicidios de mujeres son motivados por razones de género (Carcedo, 2010: 44). Siguiendo esta línea argumental, consideramos que convendría tipificar el feminicidio como delito autónomo en todos los ordenamientos jurídicos, ya que éste tiene especificidades y características propias que lo diferencian de los homicidios comunes. Defendiendo esta idea se encuentran también aquellas organizaciones de derechos humanos y feministas, que entienden que para erradicar el feminicidio es necesario establecer, como primer paso, un tipo penal fuerte que dé cuenta de la magnitud y especial brutalidad con la que se cometen estos asesinatos. ${ }^{5}$

Dado que desde la doctrina y la sociedad civil se ha planteado que el feminicidio debería ser tipificado como un tipo penal autónomo, en lo que sigue nos proponemos averiguar cuáles son dichas características.

En primer lugar, los asesinatos que constituyen feminicidio suponen actos de gran brutalidad. Se trata de muertes violentas en las que a menudo las víctimas antes de ser asesinadas son sometidas a vejaciones, lesiones y agravios que pueden constituir tortura. Esto se observa especialmente en el caso del Estado mexicano, en el que familiares o desconocidos realizan actos de gran brutalidad sobre el cuerpo de sus víctimas. ${ }^{6}$

5 Véase el trabajo de las organizaciones feminicidio.org (España) y el Observatorio Ciudadano Nacional del Feminicidio (México), su importante labor se dirige a sensibilizar a la población y las autoridades sobre los feminicidios y la violencia de género; documentar los asesinatos de mujeres a fin de crear patrones que permitan establecer acciones concretas para erradicar el feminicidio y presionar a las autoridades a fin de que se sigan las acciones necesarias para poner fin a esta problemática. En el caso del Estado mexicano las acciones del "Observatorio" han impulsado ya la tipificación del feminicidio como delito en el Código Penal Federal y en los Estados de Guerrero y Estado de México. Por su parte la organización/plataforma española feminicidio.org está cumpliendo la importante tarea de sensibilizar a la población en general y a las autoridades en especial sobre la existencia de una problemática no contemplada en la legislación española hasta el momento.

6 Un ejemplo paradigmático de esta práctica lo encontramos en el caso Campo Algodonero en el que el cuerpo de una de las víctimas, Esmeralda Herrera Monreal, apareció 
En segundo lugar, la característica más importante que define al feminicidio y que lo diferencia de otros tipos penales, como el homicidio o el asesinato, la encontramos en el mens rea presente en la conducta delictiva. Así, tal y como venimos anunciando a lo largo de este trabajo, los feminicidios son actos misóginos que encierran desigualdades estructurales de género y que subyacen en la mente del autor, por cuya causa éste realiza la conducta delictiva y justifica sus actos.

De este modo, se incardinan los feminicidios en los llamados "delitos de odio", entendiendo por éstos "los actos delictivos que una persona comete motivada por determinados perjuicios basados en la raza, etnia, edad, género, identidad de género, nacionalidad, religión, ideología o afiliación política" (Stotzer, 2007: 1). Esta categoría jurídico-penal, de reciente creación en el discurso político-criminal, ha sido objeto de numerosas críticas, que argumentan en contra de su tipificación.

En este sentido, algunos autores que han argumentado en contra de la tipificación de los delitos de odio, entendiendo que su tipificación justificaría la existencia de los prejuicios que subyacen en la comisión de estos delitos, y que además podría causar en el perpetrador una reacción contraria a la esperada con la tipificación, es decir, ésta puede desembocar en la radicalización del pensamiento del perpetrador ante la prohibición de cometer el acto y al verse perseguido por el Estado de derecho (Jacobs, 2000). Para otros, simplemente los "delitos de odio" no deben existir, ya que todos los crímenes violentos conllevan en sí mismos el desprecio del autor para con la víctima; así, si se crean categorías alternativas para juzgar los delitos en los que la víctima cumple ciertos requisitos (como puede ser el hecho de ser mujer), se deja a los acusados de estos delitos en una clara desventaja frente a los perpetradores de otros delitos violentos, vulnerando el principio de igualdad ante la ley (Anti-Defamation League, 2012).

sin rostro ni cabello, probablemente con el fin de dificultar la identificación del cuerpo; lo cual a la postre nos pude inducir a pensar — dada la pasividad de las autoridades en estos casos - que los actos tendentes a dificultar la identificación del cadáver se realizaron por terceros ajenos al perpetrador del feminicidio. No obstante, como explica Múnevar (2012: 143), sea quien sea el sujeto activo de tales actos, queda patente, con base en la brutalidad que se ejerce sobre el cuerpo de la mujer, que éste último ha sido entendido como un territorio de conquista en el que distintos amos se disputan el control del mismo y sobre el que ejercen violencias que afectan a la subjetividad del mismo con el fin de acallarlo y silenciarlo.

Esta obra está bajo una Licencia Creative Commons

Atribución-NoComercial-SinDerivar 4.0 Internacional, IIJ-UNAM.

Boletín Mexicano de Derecho Comparado, núm. 154, enero-abril de 2019, pp. 147-170. 
A este respecto, es conveniente hacer dos precisiones; en primer lugar, se ha planteado la necesidad de tipificar los delitos de odio debido a que son crímenes que afectan sobremanera a una comunidad o sector, el cual se verá fuertemente alterado psicológicamente ante la posibilidad de sufrir en su "propia piel" dichas conductas delictivas, exponiendo a los integrantes de dicha comunidad o sector a una situación de gran vulnerabilidad y desprotección jurídica. En segundo lugar, aunque conscientes de lo osadas que pueden resultar estas líneas, nos atrevemos a decir que entendemos que el mens rea que subyace en la conducta del autor del hecho delictivo podría asemejarse a aquel que pervive en la mente genocida.

Consideramos que los propósitos (la intención criminal) en los delitos de odio y en el genocidio difieren únicamente en el sujeto pasivo. No ya, en las cualidades que éste ostenta, sino en el número de sujetos contra el que se comete el hecho delictivo. Así, consideramos que el bien jurídico protegido en el crimen de genocidio es el mismo que el que se demanda proteger en el feminicidio. ${ }^{7}$

La tercera característica presente en los feminicidios y que justifica su tipificación como delito autónomo es la impunidad que rodea a estos crímenes. Las negligencias en la investigación, persecución y enjuiciamiento por parte de las autoridades mexicanas a propósito del caso Campo Algodonero y en casos análogos fueron de tal magnitud que algunas autoras han llegado a calificar al feminicidio como "crimen de Estado", por la aquiescencia imputable a los agentes gubernamentales que trabajaron en el caso (Lagarde, 2008). ${ }^{8} \mathrm{Y}$ es que la lucha de los familiares y las organizaciones de la sociedad civil por lograr justicia ha chocado frontalmente con la pa-

7 En este sentido, somos conscientes de que en el llamado derecho continental (a cuya tradición jurídica pertenecen tanto el ordenamiento jurídico español como el mexicano), la analogía en la aplicación de la pena no está permitida por la mayoría de los ordenamientos jurídicos. Sin embargo, en el ámbito legislativo podríamos no incurrir en una temeridad al expresar la posibilidad de que, fundamentando la idea en la analogía legis, pudiese interpretarse el feminicidio como un "subtipo de genocidio" que se comete de forma individualizada, caso por caso, pero que adolece de la misma intencionalidad criminal que el crimen internacional de genocidio.

8 La diputada y académica mexicana Marcela Lagarde y de los Ríos (2008) ha calificado de esa manera a los feminicidios, "son crímenes de Estado porque las instituciones encargadas de brindar seguridad han sido rebasadas y ponen en evidencia la falta de reformas políticas con perspectiva de género". 
sividad de las autoridades en algunos casos, y con la ocultación o incluso con la falsificación de información en otros. ${ }^{9}$

De esta manera, las autoridades mexicanas responsables del esclarecimiento de estos casos han contribuido a crear un clima de impunidad hacia los actos feminicidas; asimismo, han puesto de manifiesto las investigaciones realizadas por órganos jurisdiccionales o mecanismos de protección de los derechos humanos como la Comisión y la Corte Interamericana de Derechos Humanos, y el Comité para la Eliminación de todas las formas de Discriminación contra la Mujer (CEDAW).$^{10}$

9 "Las autoridades se han contradicho casi todo el tiempo. No se tiene certeza en muchos casos de que las víctimas correspondan con los cuerpos entregados y no se sabe si algunas niñas, jóvenes y mujeres desaparecidas corresponden con cuerpos sin identificar". Como afirma Lagarde (2008), durante varios años ha prevalecido la desinformación y la incertidumbre, lo que favorece la exageración y la tergiversación de datos con tono sensacionalista. Sin embargo, lo más notable y preocupante es la omisión de lo que es más evidente, la clave del asunto: el sexo de las víctimas, las cuales en una abrumadora mayoría son mujeres y niñas. Esta consideración sólo se tiene en cuenta como un dato, como si sólo se tratase de catalogar los hechos en uno de los dos sexos que conforman el ser humano, sin atender a las características contextuales en los que éstos ocurren y pasando por alto los fines opresores y el carácter misógino que subyacen en tales hechos. (Lagarde y de los Ríos, 2008).

10 Véase al respecto: CIDH. Demanda ante la Corte Interamericana de Derechos Humanos en el caso de Campo Algodonero: Claudia Ivette González, Esmeralda Herrera Monreal y Laura Berenice Ramos Mónarrez (Casos 12.496, 12.497 y 12.498) contra los Estados Unidos Mexicanos, 4 de noviembre de 2007, párr. 72 y 74; y CIDH, Situación de los Derechos Humanos de la Mujer en Ciudad Juárez, México: El Derecho a No Ser Objeto de Violencia y Discriminación, OEA/Ser. L/V/ II.117, 7 de marzo de 2003, párr. 44, anexo 1. En ambos documentos, la CIDH señala que la respuesta de las autoridades ante los crímenes ha sido notablemente deficiente, ya que el $80 \%$ de los casos permanecían impunes y además las investigaciones estaban plagadas de irregularidades y eran sumamente lentas. En la misma línea, el CEDAW destacó en un informe de 2005 que "[1] respuesta de las autoridades frente a los asesinatos, desapariciones y otras formas de violencia contra las mujeres ha sido muy deficiente, especialmente en los primeros años de los noventa y el Gobierno mismo lo reconoce que se han cometido errores e irregularidades durante ese período... Todavía, en los casos más recientes, la situación de las investigaciones, a pesar de que se ha tomado mayor conciencia de la gravedad de los hechos, no está completamente clara y se cuestiona la eficacia de la justicia”. (ONU, Comité para la Eliminación de la Discriminación Contra la Mujer, Informe de México producido por el Comité para la Eliminación de la Discriminación contra la Mujer bajo el Artículo 8 del Protocolo Facultativo de la Convención y repuesta del Gobierno de México, CEDAW/C/2005/OP.8/ MEXICO, 27 de enero de 2005, párr. 40). Véase también con relación a la impunidad de los crímenes y la negligencia del Estado de Chihuahua: ONU, Informe de la Relatora Especial sobre la violencia contra la mujer, sus causas y consecuencias, Yakin Ertürk, Integración de los Derechos Humanos de la Mujer y la Perspectiva de Género: La Violencia contra la Mujer, Misión a México, E/ CN.4/2006/61/Add.4, 13 de enero de 2006, párr. 41 y 42.

Esta obra está bajo una Licencia Creative Commons

Atribución-NoComercial-SinDerivar 4.0 Internacional, IIJ-UNAM.

Boletín Mexicano de Derecho Comparado, núm. 154, enero-abril de 2019, pp. 147-170. 


\section{MARCO JURÍDICO APLICABLE Y ESTRATEGIAS NAGIONALES PARA SU ERRADICACIÓN}

La inclusión de la categoría jurídica que tipifica el feminicidio como delito en el Código Penal Federal mexicano tuvo lugar tras la aprobación en la Cámara de Diputados y en el Senado de la Ley General de Acceso de las Mujeres a una Vida Libre de Violencia (LGAMVLV), ${ }^{11}$ en la cual se recoge dicho delito y se contemplan acciones encaminadas a erradicar la violencia contra las mujeres en el Estado, entre las que figura la llamada Alerta de Violencia de Género (AVG). ${ }^{12}$

Así, la tipificación del feminicidio como tipo penal autónomo - en el artículo 325 del Código Penal Federal mexicano - parte del tipo básico de homicidio, que se aplicará subsidiariamente cuando no se pueda acreditar que la muerte de la mujer esté motivada por las siguientes razones de género:

I. La víctima presente signos de violencia sexual de cualquier tipo; II. A la víctima se le hayan infligido lesiones o mutilaciones infamantes o degradantes, previas o posteriores a la privación de la vida o actos de necrofilia; III. Existan antecedentes o datos de cualquier tipo de violencia en el ámbito familiar, laboral o escolar, del sujeto activo en contra de la víctima; IV. Haya existido entre el activo y la víctima una relación sentimental, afectiva o de confianza; V. Existan datos que establezcan que hubo amenazas relacionadas con el hecho delictuoso, acoso o lesiones del sujeto activo en contra de la víctima; VI. La víctima haya sido incomunicada, cualquiera que sea el tiempo previo a la privación de la vida; VII. El cuerpo de la víctima sea expuesto o exhibido en un lugar público.

Por otra parte, como hemos visto, la LGAMVLV prevé la declaración de la AVG para un territorio determinado cuando se estén produciendo actos feminicidas; con el propósito de "garantizar la seguridad de éstas (mujeres), el cese de la violencia en su contra y eliminar las desigualdades producidas por una legislación que agravia sus derechos humanos".

11 Ley General de Acceso de las Mujeres a una Vida Libre de Violencia, 1o. de febrero de 2007.

12 La LGAMVLV remite al artículo 325 del Código Penal Federal en el que se define el delito de feminicidio. 
El Estado de México fue el primero en declararla en junio de 2015. ${ }^{13}$ Así, las autoridades federales del estado mexiquense - encargadas de formular la declaración en virtud del artículo 25 de la LGAMVLV - consideraron su procedencia sobre la base de la existencia de violencia feminicida, siguiendo la definición recogida en el artículo 242 bis del Código Penal del Estado de México. Este precepto - que reproduce con práctica literalidad la disposición federal sobre el feminicidio - prevé además un tipo agravado de feminicidio "cuando la víctima sea mujer menor de edad, embarazada o discapacitada, así como cuando el sujeto activo sea servidor público y haya cometido la conducta valiéndose de esta condición"; en cuyo caso la pena base de entre 40 y 70 años de prisión (o la prisión vitalicia) prevista para el delito de feminicidio, se aumentará en un tercio. ${ }^{14}$

De tal manera que el 31 de julio de 2015, ante la comisión de numerosos actos feminicidas en el Estado de México, y con el propósito de que se adoptaran medidas de emergencia que pusieran fin a tales actos ${ }^{15}$ el Sistema Nacional para Prevenir, Atender, Sancionar y Erradicar la Violencia contra las Mujeres - a través de la Secretaría de Gobernación - emitió la AGV para los municipios mexiquenses de Ecatepec, Nezahualcóyotl, Valle de Chalco, Toluca, Tlalnepantla, Naucalpan, Tultitlán, Cuautitlán Izcalli, Chimalhuacán, Ixtapaluca y Chalco. En el comunicado enviado, las autoridades del Estado de México explicaron que las medidas adoptadas se organizarían en torno a tres ejes de acción: prevención de la violencia de género, seguridad y acceso a la justicia para las mujeres.

Con relación al eje denominado "prevención de la violencia de género", el comunicado señaló la realización de talleres sobre violencia de género y sexualidad en los niveles educativos de primaria y secundaria; así como, una campaña de sensibilización y concienciación y "se estará en plena alerta $[$ sic $]$ para detectar si alguna niña o joven esté sufriendo alguna situación de violencia".

13 El estado mexiquense tiene una de las tasas más altas de feminicidios desde la década de los noventa (ONU Mujeres, 2012) (Incháustegui y López, 2012).

14 Código Penal del Estado de México, libro segundo, título tercero. Delitos contra las personas. Subtítulo primero. Delitos contra la vida y la integridad corporal. Capítulo II, artículo 242 bis, en vigor desde el 30 de abril de 2013.

15 Véase al respecto la definición de AVG recogida en el artículo 22 LGAMVLV: "conjunto de acciones gubernamentales de emergencia para enfrentar y erradicar la violencia feminicida en un territorio determinado, ya sea ésta ejercida por individuos o por la propia comunidad" (énfasis añadido).

Esta obra está bajo una Licencia Creative Commons

Atribución-NoComercial-SinDerivar 4.0 Internacional, IIJ-UNAM.

Boletín Mexicano de Derecho Comparado, núm. 154, enero-abril de 2019, pp. 147-170. 
No entendemos en qué se traduce el vocablo "en plena alerta", pero sobre todo quién va a estarlo y hasta cuándo, consideramos esta medida ambigua y vacía de contenido, máxime teniendo en cuenta que ésta debería ser una medida de emergencia, según lo dispuesto en el artículo 22 de la LGAMVLV. Respecto al eje "seguridad", se dispone en el comunicado de AVG que, en los municipios del Estado de México para los que fue emitida la declaración, se integrarán Células de Reacción Inmediata para atender llamadas de emergencia y se elaborarán protocolos de actuación y reacción institucional para la localización de niñas y mujeres desaparecidas, acción en la que participará el Sistema Nacional Integral de Protección de la Familia (DIF) estatal y local. En lo que se refiere al eje "acceso a la justicia", el secretario general de gobierno se limitó a informar de que en los últimos años se han puesto en marcha estrategias para combatir la violencia contra las mujeres. Ciertamente, son muchas las medidas tomadas sobre este particular, ${ }^{16}$ aunque éstas no son medidas de emergencia ni se tomaron a propósito de la declaración de AVG. ${ }^{17}$

16 A saber, la tipificación en el Código Penal (estatal y federal) del delito de feminicidio; la creación de la Subprocuraduría de Delitos Vinculados a la Violencia de Género y las fiscalías especializadas en Feminicidios, Trata de Personas y para la Investigación de Personas Desaparecidas, No Localizadas, Ausentes o Extraviadas. Además, el secretario expresó que a lo largo de los últimos años se ha consolidado el marco jurídico en la materia, por medio de la adopción de las leyes de "Acceso de las Mujeres a una Vida Libre de Violencia", la "Ley para Prevenir, Atender, Combatir y Erradicar la Trata de Personas" y la "Ley para la Protección y Asistencia a Víctimas"; se ha puesto en marcha la línea 01 800 TE AYUDA, y desde septiembre de 2014 se han implementado desde la Procuraduría General de Justicia del Estado de México (PGJEM) más de 9,000 acciones de protección a favor de las mujeres y más de 82,000 asistencias gratuitas, entre las que se cuenta el apoyo psicológico, jurídico y la atención médica.

17 En este sentido, el 22 de septiembre de 2015, en el marco de la "Segunda Sesión Ordinaria de la Comisión Temporal de Seguridad del Sistema en el Marco de la Alerta de Violencia de Género", se presentó al Grupo Especializado en Atención a la Violencia Familiar y de Género de Toluca (GEAVFyG) como modelo de éxito en la implementación de las medidas comprendidas en la AVG. Además, se apeló a la aplicación de estas medidas por los otros diez municipios comprendidos dentro de la AVG, a los cuales se les formularon críticas en relación a su inactividad al respecto (Gobierno del Estado de México, 2015, Alerta de Género (última visita: noviembre de 2015). Ahora bien, lo que no se explicó es que el GEAVFyG no había sido creado ex profeso, sino que se estableció en el año 2013, integrado por doce efectivos capacitados en asuntos de violencia de género que ofrecen asistencia psicológica, jurídica, social y médica. Es decir, se trata de un grupo permanente que trabaja en el campo de acción de la violencia de género, pero no constituye una medida de emergencia en el sentido previsto en la LGAMVLV.

Esta obra está bajo una Licencia Creative Commons Atribución-NoComercial-SinDerivar 4.0 Internacional, IIJ-UNAM. Boletín Mexicano de Derecho Comparado, núm. 154, enero-abril de 2019, pp. 147-170. 
Asimismo, las entidades mexiquenses consultadas (Universidad Autónoma del Estado de México; Comisión de Derechos Humanos del Estado de México, y Centro de Investigación, Capacitación, Estudios y Proyectos Especializados en Género) manifestaron que en términos generales las medidas propuestas son inefectivas por inaplicadas. Cuestión que atribuyen a la ausencia de información sobre el contenido específico de las medidas que la componen. En este sentido, se ha de destacar que tras varios años desde la declaración de la AVG, todavía no se ha concretado el contenido de dichas medidas de emergencia. ${ }^{18}$

\section{Otras medidas y estrategias no comprendidas en la Alerta de Violencia de Género}

$\mathrm{Al}$ margen de la creación de medidas específicas que predispone la declaración de la AVG, el Plan "Vida sin Violencia" de Inmujeres" —elaborado conforme a los pilares de prevención, atención, sanción y erradicación previstos en el artículo 6o. del Reglamento de la Ley General de Acceso de las Mujeres a Una Vida Libre de Violencia ${ }^{20}$ - prevé la adopción de

18 En el caso concreto de Toluca, capital del Estado de México, se ha constatado que la celeridad en la fijación de acciones urgentes no ha sido una prioridad para las autoridades gubernamentales. Así, un mes después de dicha declaración, tuvo lugar la Séptima Sesión de Trabajo del Sistema Municipal para la Igualdad de Trato y Oportunidades entre hombres y mujeres, en ella representantes de Inmujeres, la institución que coordina el cumplimiento de la política nacional en materia de igualdad sustantiva y coadyuva a erradicar la violencia contra las mujeres, expresaron la necesidad de que en un breve espacio de tiempo se diese cuenta de las acciones que se están llevando a cabo en relación a la Alerta de Género.

19 Es importante destacar que este programa conlleva la gestión y coordinación de este, por diversas secretarias (equivalentes a los ministerios españoles). De esta manera, es un trabajo interdisciplinar en el que se desempeñan entre otras la Secretaría de Seguridad Pública, Secretaría de Educación y la Suprema Corte de Justicia de la Nación.

20 Reglamento a la Ley General de Acceso de las Mujeres a una Vida Libre de Violencia, en vigor desde 11 de marzo de 2008. Entre las medidas citadas destacan, en el ámbito de prevención: los talleres de prevención de la violencia familiar; el Manual de buenas prácticas para investigar y sancionar el acoso laboral y/o el acoso sexual de 2013, emitido por la Suprema Corte de Justicia de la Nación (instrumento de vital importancia ya que permite adoptar un criterio común en los casos de acoso laboral y/o sexual que entren dentro de la competencia material de la Suprema Corte de Justicia de la Nación, órgano paralelo al Tribunal Constitucional español, que como órgano superior de justicia constitucional mexicano ostenta el privilegio de que sus resoluciones no pueden ser objeto de recurso, SCJN, 2013); y el Manual de prevención de la violencia de género en diversos contextos de 2012, emitido por la Secretaría de Seguridad Pública (un instrumento creado con el fin de ser utilizado como mecanismo para sensibilizar a la población sobre cuestiones de género, con el fin de que identifiquen los casos de violencia, SSP, 2012).

Esta obra está bajo una Licencia Creative Commons

Atribución-NoComercial-SinDerivar 4.0 Internacional, IIJ-UNAM.

Boletín Mexicano de Derecho Comparado, núm. 154, enero-abril de 2019, pp. 147-170. 
un conjunto de medidas y estrategias destinadas a hacer frente a los feminicidios siguiendo los pilares mencionados.

En este sentido, destaca - a nivel federal y dentro del pilar prevención - el "Programa de reeducación para víctimas y agresores de violencia de pareja", desarrollado por el Instituto Nacional de Salud Pública con el propósito de "crear redes de atención coordinando la acción de diversas instituciones con miras a efectuar adecuadas derivaciones y por consiguiente la necesaria intervención tanto para víctimas como para agresores", al objeto de construir relaciones afectivas en un plano de igualdad entre hombre y mujer, y así dar cumplimiento a la LGAMVLV.

En relación al pilar sanción, cabe mencionar la adopción del "Protocolo de investigación ministerial, policial y pericial con perspectiva de género para el delito de feminicidio", y del "Protocolo de investigación ministerial, policial y pericial con perspectiva de género para la violencia sexual" (PGR, 2012); y en especial del Protocolo para juzgar con perspectiva de género de la Suprema Corte de Justicia de la Nación: haciendo realidad el derecho a la igualdad (SCJN, 2013, Protocolo para juzgar con perspectiva de género). Instrumento de vital importancia para hacer efectiva la rendición de cuentas en casos de feminicidio, pues fue creado con el propósito de

...hacer frente a las problemáticas detectadas y las medidas de reparación ordenadas por la Gorte Interamericana de Derechos Humanos en los casos "Campo Algodonero", Inés Fernández Ortega (CIDH, 2010, caso Fernández Ortega y otros vs. México) y Valentina Rosendo Cantú (CIDH, 2010, caso Rosendo y otra vs. México), relativas al ejercicio del control de convencionalidad por quienes imparten justicia y, por tanto, a la aplicación del derecho de origen internacional, así como al establecimiento de instrumentos y estrategias de capacitación y formación de perspectiva de género y derechos de las mujeres (SGJN, 2013: 8).

En lo que respecta al Estado de México llama la atención la ausencia de programas especializados para dar cumplimiento a los pilares de la prevención y sanción del programa federal "Vida sin Violencia", a diferencia de lo que ocurre en otros estados mexicanos que sí cuentan con programas de prevención (Sonora y Veracruz) o relativos a la sanción (Guerrero y Oaxaca). Con relación a la atención, el Estado de México tiene un programa denominado "Modelo de atención a mujeres en situación de 
violencia, sus hijas e hijos" de 2012, emitido por el Consejo Estatal de la Mujer y Bienestar Social. ${ }^{21}$

De esta manera, aunque tanto en el ámbito federal como en el estatal y local se están dando pasos hacia la erradicación de la violencia contra la mujer en general, y la comisión de feminicidios en particular, es necesario incrementar esfuerzos para lograr - tras su tipificación como delitola aplicación integra de la LGAMVLV. Especialmente, convendría que se concretaran las medidas de emergencia que comprenden la AGV declarada en el Estado de México y se adoptaran los recursos necesarios para su posterior ejecución, así como la elaboración de programas de prevención y sanción en el Estado de México al amparo del plan "Vida sin Violencia”, sobre todo en el ámbito local, pues, sólo así, se enviaría a la sociedad el mensaje de que la violencia contra la mujer no está permitida y que su comisión implica la condena del autor.

En lo que respecta a España la diferencia más significativa deviene del hecho de que en el ordenamiento jurídico español el delito de feminicidio no está tipificado como delito autónomo. El legislador español no ha prestado atención a esta cuestión ni siquiera en la reciente reforma del Código Penal del año 2015 (CP), ${ }^{22}$ perdiendo la oportunidad de comprometerse seriamente con la lucha contra la perpetración de actos feminicidas a pesar del aumento de la criminalidad respecto a este tipo delictivo en los últimos años. En su lugar, se han realizado ciertas modificaciones en los delitos de asesinato y homicidio que estudiaremos a continuación.

Aunque, en un primer momento podría parecer que la legislación española, fundamentalmente por medio de la Ley Orgánica de Medidas de Protección Integral contra la Violencia de Género (LIVG), ${ }^{23}$ cubre todos los aspectos derivados de este tipo de violencia, y por ende también el feminicidio, la realidad es diametralmente opuesta. Conforme se dispone en la Exposición de Motivos de la LIVG, por violencia de género se entenderá aquella que "se dirige sobre las mujeres por el hecho mismo de serlo, por

21 Este programa se limita a detallar las consecuencias psicosociales que presentan las mujeres que han sufrido violencia de género, a hacer acopio de la normativa nacional e internacional con relación al tema y por último a detallar las instituciones mexiquenses que trabajan en estas cuestiones.

22 Ley Orgánica 1/2015, del 30 de marzo, por la que se modifica la Ley Orgánica 10/1995, del 23 de noviembre del Código Penal.

23 Ley Orgánica 1/2004, del 28 de diciembre, de Medidas de Protección Integral contra la Violencia de Género.

Esta obra está bajo una Licencia Creative Commons

Atribución-NoComercial-SinDerivar 4.0 Internacional, IIJ-UNAM.

Boletín Mexicano de Derecho Comparado, núm. 154, enero-abril de 2019, pp. 147-170. 
ser consideradas, por sus agresores, carentes de los derechos mismos de libertad, respeto y capacidad de decisión". En este sentido, aunque esta definición concuerda con los elementos del feminicidio, hay que precisar que - en virtud del artículo 1o. de la LIVG - se requiere que el sujeto activo del delito (hombre) y el pasivo (mujer) "mantengan o hayan mantenido una relación de afectividad, aún sin convivencia".

Llegados a este punto, la pregunta que nos planteamos es: ¿qué calificación jurídica recibe en España la muerte de una mujer por razones de género si entre la víctima y el perpetrador no existe ni ha existido relación de afectividad?

El homicidio se regula en el artículo 138.1 del CP, que reza así: "el que matare a otro será castigado, como reo de homicidio, con la pena de prisión de diez a quince años". Tras la reforma de 2015, se prevé un tipo cualificado de homicidio - en cuyo caso los hechos serán castigados con la pena superior en grado, es decir de 15 a 22 años y medio - cuando concurran alguno de los requisitos del artículo 140.1 CP; entre ellos: "que el hecho fuera subsiguiente a un delito contra la libertad sexual que el autor hubiera cometido sobre la víctima".

Pues bien, como hemos visto con anterioridad - y así lo recoge la legislación mexicana - es habitual que los feminicidios sean precedidos de un delito contra la libertad sexual, generalmente violación y otros actos que atentan gravemente contra la dignidad de las mujeres (Agatón n.d.). De este modo, el feminicidio podría ser castigado en España por la vía del citado precepto. Incluso, en ciertos casos el feminicidio podría encajar en el tipo delictivo asesinato (artículo 139 CP) siempre que la brutalidad con la que se cometiesen los actos feminicidas encajara en el elemento "ensañamiento"24 — también alevosía o mediando precio o recompensa - o si se perpetrase "para evitar que se descubra la comisión de otro delito" (139.1. 4o. CP); en cuyo caso se le impondría al perpetrador de actos de violencia sexual y asesinato una pena de 20 a 25 años. Por último, es posible que el asesinato no hubiese sido cometido con el fin de encubrir el acto de violencia sexual, sino que el mens rea del autor hubiese sido la comisión de ambos actos de forma dolosa, independiente y cons-

24 El criterio jurisprudencial seguido por el Tribunal Supremo respecto al ensañamiento define este elemento del asesinato como "la disposición en la ejecución del acto que pretenda aumentar deliberada e intencionalmente el dolor del ofendido". Véase: STS, del 6 de octubre de 2015, núm. 573/2015. 
ciente, en cuyo caso en virtud del artículo 140. 1. 2o CP, sería castigado con la pena de prisión permanente revisable.

A la vista de esta última posibilidad, los actos feminicidas en España pueden ser castigados con una pena mayor que en México ("prisión permanente revisable"), si bien el condenado por tales actos en España no lo sería por la comisión del delito de feminicidio, dada la ausencia de disposiciones legales en las que se recoja expresamente el tipo delictivo.

En este sentido, y dado que los actos de feminicidio pueden ser castigados en España por la vía de los citados preceptos del CP, la pregunta que sigue es: ¿es necesario tipificarlo como delito autónomo?

En nuestra opinión, la respuesta no está clara. En primer lugar, cabe considerar, como así se expuso en el primer epígrafe, que el feminicidio es un tipo penal cuya tipificación obedece al hecho de que el autor mata a la mujer por razones de género. En este sentido, ninguno de los preceptos del $\mathrm{CP}$ que hemos mencionado se refiere expresamente a este particular, pasando por alto el carácter discriminatorio y misógino que subyace en la violencia feminicida, y la estructura patriarcal que alienta y perpetúa las relaciones desiguales entre hombre y mujer. Si bien, el CP recoge como agravante el hecho de cometer el acto delictivo por "razones de género" (artículo 22.4 CP). En consecuencia, entraría en este caso la muerte de una mujer por razones de género, aunque la víctima no tuviese o hubiese tenido una relación o vínculo afectivo-sexual o íntimo con el agresor. Se incluirían así los supuestos de "una mujer que sufre una agresión sexual por parte de un extraño y luego es asesinada por éste. También entra en esta tipología el caso del vecino que mata a su vecina por misoginia u otras razones de género". ${ }^{25}$

25 En este sentido, aunque en España siguen siendo mayoría los casos de feminicidio íntimo, lo cierto es que a lo largo de 2015 aumentaron los casos de feminicidios no íntimos, como parecen ser los casos de Laura Hoyo, de 24 años (Cuenca), asesinada brutalmente por el ex novio de su amiga y Denise Pikka Thiem, la peregrina estadounidense asesinada mientras hacia el Camino de Santiago. En ambos casos, aunque la investigación policial realizada hasta el momento sobre el móvil de los crímenes no ha dado lugar a que podamos afirmar con rotundidad que se trata de feminicidios no íntimos, todo apunta a que en ambos casos la misoginia tuvo un papel fundamental en el mens rea de los asesinos. En el primero de los casos, la calificación de feminicidio de Marina Okarynska, de 26 años, está clara, la muerte de Laura del Hoyo podría ser calificada de feminicidio no íntimo si se comprueba que el asesino la mató por razones de género. Por su parte, según las últimas averiguaciones en el caso Denise, parece ser que el asesino confeso "tenía algunos antecedentes" y "había molestado a mujeres que habían pasado por la zona", por lo que todo parece indicar que las razones de género están presentes en la comisión de este crimen (DL 2015).

Esta obra está bajo una Licencia Creative Commons

Atribución-NoComercial-SinDerivar 4.0 Internacional, IIJ-UNAM.

Boletín Mexicano de Derecho Comparado, núm. 154, enero-abril de 2019, pp. 147-170. 
No obstante, como venimos diciendo, el artículo lo. de la LIVG impide que sean considerados al objeto de dicha norma aquellos casos en los que no hay una relación afectiva entre el hombre y la mujer. En este sentido, se ha de recordar que esta ley fue creada como un instrumento para, inter alia, fortalecer el marco jurídico penal y procesal; con el fin de asegurar una protección integral de las víctimas y garantizarles derechos en los ámbitos laboral y económico, posibilitar la capacitación de profesionales especializados y reconocer también como víctimas a los hijos e hijas de las mujeres víctimas de violencia de género.

En términos generales, la gran mayoría de las disposiciones previstas en esta ley se han cumplido. Sin embargo, la protección de la que gozan las víctimas de violencia de género en virtud de la LIVG no se extiende a aquellos feminicidios en los que autor y víctima no hubiesen mantenido relación de afectividad. En consecuencia, la tipificación del feminicidio como tipo delictivo autónomo se justificaría en la necesidad de crear medidas y estrategias específicas destinadas a proteger a todas las víctimas de feminicidio, independientemente de la relación que éstas tuviesen con el agresor.

\section{CONCLUSiOnes}

Sobre la base de argumentación anteriormente expuesta, consideramos pertinente relatar las siguientes conclusiones, las cuales en su mayoría son recomendaciones que consideramos que ambos países deben tomar en cuenta para poner fin a la comisión de actos feminicidas.

Con relación al caso mexicano, y dado que el feminicidio ya está considerado como tipo penal autónomo en el ámbito federal y, en algunos casos, estatal, y teniendo en cuenta que su tipificación es lo suficientemente exhaustiva; podemos considerar que las razones por las que se siguen cometiendo se escapan a la lógica de la criminalización. Así, a riesgo de ser excesivamente escuetos consideramos que los principales motivos por los que tienen lugar los feminicidios en México comprenden la desigualdad estructural que pervive en la sociedad mexicana, a lo que se añade una fuerte cultura patriarcal que fomenta la existencia de sentimientos de posesión en los hombres hacia las mujeres; la escasez e ineficacia de políticas públicas, medidas y estrategias destinadas a promover la igualdad de 
género, y la impunidad coyuntural existente en el país que impide llevar a cabo investigaciones y enjuiciamientos prolijos y eficientes.

En virtud de lo cual, queremos formular las siguientes recomendaciones:

a) El gobierno federal, así como los gobiernos estatales deben incidir en la aplicación integral de la LGAMVLV y además reforzar la creación y ejecución de medidas, estrategias y políticas públicas dirigidas a educar a la sociedad en igualdad. Dichas acciones deberían tomarse tanto a nivel escolar, en todos los niveles educativos, como a nivel comunitario, en este caso especialmente en municipios que cuentan con altos niveles de violencia contra la mujer. En este caso no se trata de reforzar la normativa sino de hacerla efectiva a través de acciones concretas, incluyendo la creación de mecanismos objetivos e independientes de monitoreo y evaluación.

b) La investigación policial, institucional y judicial de los feminicidios debe ser reforzada; en este sentido es necesaria la capacitación en género de los encargados de esclarecer y sancionar los feminicidios.

c) En el caso del Estado de México, las medidas que componen la AVG deben ser concretadas y ejecutadas con urgencia. Asimismo, se debe considerar la ampliación de la declaración a otros municipios del Estado, y a cuentas entidades estatales requieran de la adopción de esta medida.

Con relación a España, consideramos que debería (re)abrirse un debate a nivel parlamentario y social con el fin de abordar la tipificación del feminicidio. En este sentido, se ha descargado demasiada responsabilidad sobre la LIVG y falta reabrir el foro de discusión para comprender que "violencia de género" y "feminicidio" no son sinónimos. Es necesario ser consciente de que su equiparación está permitiendo que muchas asesinadas, que no tenían o habían tenido una relación afectiva con su asesino, queden en desventaja respecto a las que sí, no siendo además consideradas a todos los efectos como víctimas de un delito (el feminicidio) que cuenta con características propias que lo diferencian de los asesinatos y homicidios.

Respecto a lo cual se formulan las siguientes recomendaciones: 
a) Convendría tipificar en el CP el feminicidio como tipo penal autónomo, y revisar la LIVG a fin de incluir a las víctimas de este delito o sus causahabientes entre las víctimas de violencia de género, que quedarían protegidas por las medidas procedimentales y sancionatorias específicas para este tipo de violencia que se disponen en la citada ley.

b) En consecuencia, las políticas públicas, estrategias y medidas dirigidas a desarrollar y aplicar la legislación en materia de lucha contra la violencia de género deberían incluir disposiciones específicas para prevenir los feminicidios.

\section{BIBLIOGRAFÍA}

AGATÓN, Isabel, 2013, "El «feminicidio» se puede prevenir y se debe evitar". Disponible en: http://wwre.mujeresenred.net/spip.php?article2097.

Anti-Defamation League, 2012, Constitutional Challenges to Hate Crimes Statutes. Disponible en: http://wrwr.adl.org/assets/pdf/combating-hate/Hate-Crimes-Law.pdf.

Carcedo, Ana, 2010, "No olvidamos ni aceptamos: feminicidio en centroamérica 2000-2006".

Fernández de JuAn, Teresa, 2004, Violencia contra la mujer en México, Ciudad de México, Comisión Nacional de los Derechos Humanos de México.

Hester, Marianne, 2006, "La brujo-manía en Inglaterra en los siglos XVI y XVII como control social de las mujeres (The Witch-Craze in Sixteenth and Seventeenth Century, England as Social Control of Women)", en Russell, D. y RADFORD, J. (eds.), Feminicidio: la poli (Femicide: The Politics of Women Killing), Nueva York, Twayne.

Jacobs, James B. y Potter, Kimberly, 2000, Hate Crimes: Criminal Law and Identity Politics, Nueva York, Oxford University Press.

LAGARDE Y DE LOS RíOS, Marcela, 2008, "Antropología, Feminismo y política: violencia feminicida y derechos humanos de las mujeres", Retos teóricos y nuevas prácticas.

Livingston, Jessica, 2004, "Murder in Juárez: Gender, Sexual Violence and the Global Assembly Line", Frontiers: A Journal of Women Studies. 
MacFarlane, Alan, 1970, Witchcraft in Tudor and Stuart England: A Regional and Comparative Study, Londres, Routledge.

Mónarrez Fragoso, Julia, 2002, "Debate feminista", Feminicidio sexual serial en Ciudad fuárez: 1993-2001, México.

Múnevar M., Dora Inés, 2012, "Delito de feminicidio. Muerte violenta de mujeres por razones de género", Revista de Estudios Socio-furídicos.

PantaleO, Katherine, 2010, "Gendered Violence: An Analysis of the Maquiladora Muerders", International Criminal Justice Review.

Russell, Diana, 2006, "Definición del feminicidio y conceptos relacionados", s. d.

Stotzer, Rebecca, 2007, "Comparision of Hate Crime Rates AcrossProtected and Unprotected Groups", s. d.

Trevor-Roper, Redwald H., 1969, The European Witch-Craze of the Sixteenth and Seventeenth Centuries, Penguin, Harmondsworth.

\section{Documentos Naciones Unidas}

Comité Para la Eliminación de la Discriminación Contra la Mujer, 1992, Recomendación General núm. 19, del 29 de enero de 1992.

Comité Para la Eliminación de la Discriminación Contra La MuJer, 2005, Informe de México producido por el Comité para la Eliminación de la Discriminación contra la Mujer bajo el Artículo 8o. del Protocolo Facultativo de la Convención y repuesta del Gobierno de México, CEDAW/C/2005/OP.8/ MEXICO, del 27 de enero de 2005.

Consejo de Derechos Humanos de las Naciones Unidas, Informe del Grupo de Trabajo en la cuestión de la discriminación contra la mujer en la ley y en la práctica, Grupo de Trabajo sobre la cuestión de la Discriminación contra la Mujer en la Legislación y en la práctica del Consejo de derechos humanos de Naciones Unidas, A/HRG/29/40/Add.6, del 17 de junio de 2015.

ONU, GOMITÉ PARA LA Eliminación DE LA DisGriminación CONTRA LA MuJer, 2005, Informe de México producido por el Comité para la Eliminación de la Discriminación contra la Mujer bajo el Artículo 8 del Protocolo Facultativo de la Convención y repuesta del Gobierno de México, CEDAW/C/2005/ OP.8/MEXICO. 27 de enero de 2005.

Esta obra está bajo una Licencia Creative Commons

Atribución-NoComercial-SinDerivar 4.0 Internacional, IIJ-UNAM.

Boletín Mexicano de Derecho Comparado, núm. 154, enero-abril de 2019, pp. 147-170. 
ONU Mujeres, 2012, Violencia feminicida en México. Caracteristicas, Tendencias y nuevas expresiones en las entidades federativas 1985-2010, Instituto Nacional de las Mujeres México, LXI Legislatura Cámara de Diputados, Comisión Especial para conocer y dar Seguimiento Puntual y Exhaustivo a las Acciones que han Emprendido las Autoridades Competentes con Relación a los Feminicidios Registrados en México, México.

ONU, CONSEJO ECONÓMICO Y SOCIAL, Integración de los derechos humanos de la mujer y la perspectiva de género: la violencia contra la mujer, Informe de la Relatora Especial sobre la violencia contra la mujer, sus causas y consecuencias, Takin Ertürk, Misión a México, E/CN.4/2006/61/Add.4, 13 de enero de 2006.

Corte Interamericana de Derechos Humanos, caso Fernández Ortega y otros vs. México, sentencia del 30 de agosto de 2010.

Corte Interamericana De Derechos Humanos, caso Rosendo y otra vs. México, sentencia del 31 de agosto de 2010.

Comisión Interamericana de Derechos Humanos, Demanda ante la Corte Interamericana de Derechos Humanos en el caso de Campo Algodonero: Claudia Ivette González, Esmeralda Herrera Monreal y Laura Berenice Ramos Mónarrez (casos 12.496, 12.497 y 12.498) contra los Estados Unidos Mexicanos, del 4 de noviembre de 2007.

Comisión Interamericana De Derechos Humanos, Situación de los derechos humanos de la mujer en Ciudad fuárez, México: El derecho a no ser objeto de violencia y discriminación, OEA/ Ser. L/V/II.117, 7 de marzo de 2003, anexo 1.

\section{Otros documentos mexicanos}

Gámara de Diputados de la República, 2010, Programa de reeducación para víctimas y agresores de violencia de pareja, México.

Consejo Estatal de la Mujer y Bienestar Social, 2012, Modelo de atención a mujeres en situación de violencia, sus hijos e hïas, México.

Gobierno Del Estado De México, 2015, Alerta de Género, Toluca, disponible en: http://alertadegenero.edomex.gob.mx/.

Gobierno Del Estado De MéXico, Programa, Modelo de atención a mujeres en situación de violencia, sus hijas e hijos, 2012, Consejo Estatal de la Mujer y Bienestar Social 
Esta revista forma parte del acervo de la Biblioteca Jurídica Virtual del Instituto de Investigaciones Jurídicas de la UNAM

Procuraduría General de la República, 2012a, Protocolo de investigación ministerial, policialy pericial con perspectiva de género para el delito de feminicidio, México, Fevimtra.

Procuraduría General de la República, 2012b, Protocolo de investigación ministerial, policial y pericial con perspectiva de género para la violencia sexual, México.

Secretaría De SEguridad PúBlica, 2012, Manual de prevención de la violencia de género en diversos contextos, México.

Suprema Corte de Justiaia de LA NAGión, 2013, Manual de buenas prácticas para investigar y sancionar el acoso laboral y/o el acoso sexual, México.

SuPrema Corte de Justiaia de la NACión, 2013, Protocolo para juzgar con perspectiva de género de la Suprema Corte de Justicia de la Nación: haciendo realidad el derecho a la igualdad, México. 